\title{
Nephrogenesis in kidney organoids derived from human pluripotent stem cells
}

$\mathrm{N}$ ew research, published in Nature, describes the generation of kidney organoids from human pluripotent stem cells (hPSCs). These organoids contained multiple nephron segments surrounded by renal interstitium and endothelial cells. "This is the first time a kidney organoid has been generated that contains all of the renal components, including segmented nephrons, collecting ducts, a surrounding interstitium and a vascular network," says first author on the paper, Minoru Takasato. "Considering that the kidney is the organ responsible for blood filtration, the generation of renal vasculature is a noteworthy step forward in recreating a functional kidney in vitro and for future applications in regenerative medicine."

The researchers had previously demonstrated the ability to simultaneously

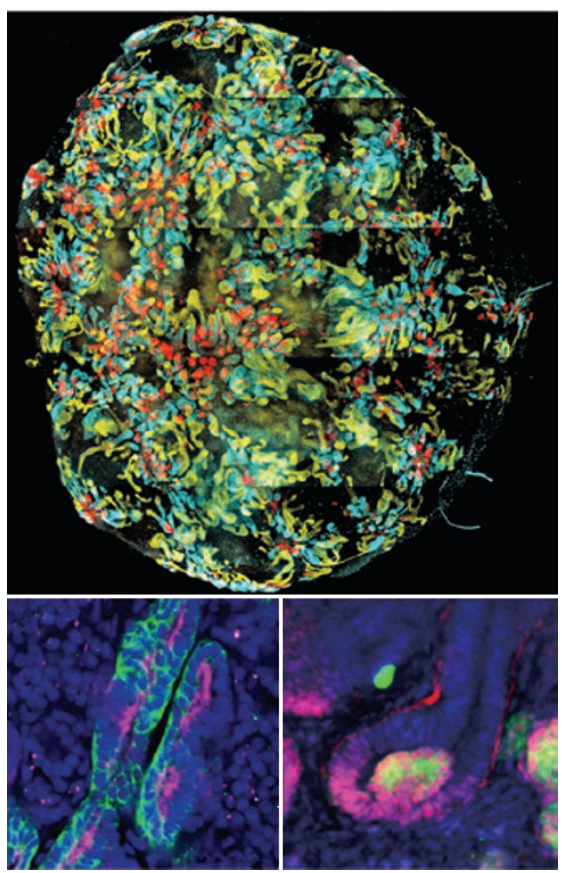

Kidney organoids were structurally complex (top) and contained nephron segments such as loops of Henle (bottom left) and glomeruli (bottom right). Permission obtained from Nature Publishing Group ( ) Takasato, M. et al. Nature doi:10.1038/nature15695. generate collecting duct and nephron progenitors from hPSCs, which was noteworthy as these cells have distinct temporospatial origins despite both being derived from the intermediate mesoderm. The ureteric epithelium, from which the collecting duct is derived, arises from the anterior intermediate mesoderm, whereas the metanephric mesenchyme, from which nephrons are derived, arises from the posterior intermediate mesoderm. The researchers have now shown that they can direct the extent of ureteric epithelium and metanephric mesenchyme formation from hPSCs by altering the levels and timing of Wnt and FGF9 exposure. "Our previous protocol was not optimal in that we were getting too much of one cell type and not enough of the other," explains lead researcher Melissa Little. "In this study, using our understanding of embryology in the mouse, we found a way to 'reposition' the state of development of the cells so we got all of the right progenitors at the same time."

Using their revised protocol, the researchers induced kidney organoid cultures derived from hPSCs to undergo nephrogenesis, with the derived nephrons segmenting into four components: the collecting duct, early distal tubule, early proximal tubule and the glomerulus. The organoids showed complex morphogenetic patterning, with collecting duct trees connecting to distal and proximal tubules, and glomeruli at the top of each organoid. In addition, the researchers found evidence of collecting ducts, which are derived from the ureteric epithelium. By day 18 of culture, distinct epithelial subtypes were evident, including early glomeruli comprising a Bowman capsule connected to proximal tubules, and foot processes characteristic of podocytes, as assessed by transmission electron microscopy. The researchers also found evidence of perivascular cells lying alongside endothelia and early mesangial cells invaginating glomeruli, as well as evidence of endothelial invasion into glomeruli. "This work represents the culmination of many years of research that came from a desire to use our basic understanding of kidney development to regenerate a kidney," says Little. "Now we have a kidney organoid that has collecting ducts, but also nephrons that are patterning into different segments, a surrounding interstitium, and forming blood vessels."

To investigate the maturity of their kidney organoids the researchers compared the transcriptional profiles of their organoids with those of human fetuses. Kidney organoids cultured for 11 or 18 days had comparable transcriptional profiles to first trimester fetal kidneys, whereas less developed organoids had more primitive transcriptional profiles. In addition, Little and colleagues demonstrated the ability of proximal tubules to endocytose dextran and differentially undergo apoptosis in response to the nephrotoxin cisplatin, demonstrating the functional maturation of the organoids.

The researchers say that short and long term possibilities exist for future work. "In the short term, we would like to see if an organoid like this will be useful for screening drugs for toxicity to the kidney and we are also generating stem cells from patients with genetic kidney disease so that we can use this approach to model and study their disease," says Little.

"However, in the longer term, what we have generated is all the cells you need to make a much bigger organ so there may be options for bioengineering a replacement organ or cellular therapy."

Susan J. Allison

Original article: Takasato, M. et al. Kidney organoids from human iPS cells contain multiple lineages and model human nephrogenesis. Nature doi:10.1038/nature15695 